\title{
Photoresponse of Graphene-Gated Graphene-GaSe Heterojunction Devices
}

Wonjae Kim, ${ }^{\dagger}$ Sanna Arpiainen, ${ }^{* \dagger}{ }^{\dagger}$ Hui Xue, ${ }^{\ddagger}$ Miika Soikkeli, ${ }^{\dagger}$ Mei Qi, ${ }^{\ddagger}$ Zhipei Sun, ${ }^{\ddagger}, \perp \odot$

Harri Lipsanen, ${ }^{\ddagger}$ Ferney A. Chaves, ${ }^{\S}$ David Jiménez, ${ }^{\S}$ and Mika Prunnila ${ }^{\dagger, \perp}$

${ }^{\dagger}$ VTT Technical Research Center of Finland Ltd., P.O. Box 1000, FI-02044 VTT, Finland

${ }^{\ddagger}$ Department of Electronics and Nanoengineering, Aalto University, Tietotie 3, Espoo 02150, Finland

${ }^{\S}$ Department d'Enginyeria Electrònica, Escola d'Enginyeria, Universitat Autònoma de Barcelona, Campus UAB, Bellaterra E-08193, Spain

${ }^{\perp}$ QTF Centre of Excellence, Department of Applied Physics, Aalto University, Aalto FI-00076, Finland

Supporting Information

ABSTRACT: Because of their extraordinary physical properties, low-dimensional materials including graphene and gallium selenide $(\mathrm{GaSe})$ are promising for future electronic and optoelectronic applications, particularly in transparent-flexible photodetectors. Currently, the photodetectors working at the near-infrared spectral range are highly indispensable in optical communications. However, the current photodetector architectures are typically complex, and it is normally difficult to control the architecture parameters. Here, we report graphene-GaSe heterojunction-based field-effect transistors with broadband photodetection from 730-1550 $\mathrm{nm}$. Chemical-vapor-deposited
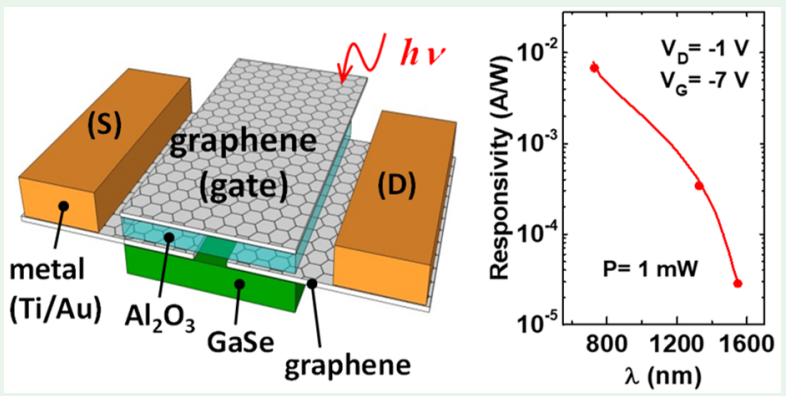
graphene was employed as transparent gate and contact electrodes with tunable resistance, which enables effective photocurrent generation in the heterojunctions. The photoresponsivity was shown from 10 to $0.05 \mathrm{~mA} / \mathrm{W}$ in the near-infrared region under the gate control. To understand behavior of the transistor, we analyzed the results via simulation performed using a model for the gate-tunable graphene-semiconductor heterojunction where possible Fermi level pinning effect is considered.

KEYWORDS: graphene, GaSe, heterojunction, Schottky, photodetector

\section{INTRODUCTION}

Properties of layered bulk semiconducting materials, including III-VI binary compounds like GaSe, have been identified and well-understood since the 1960s. ${ }^{1,2}$ Recently, interest in various two-dimensional (2D) layered semiconductors has risen with inspiration given by graphene, ${ }^{3,4}$ as they can be exploited as active materials for a large range of applications. As the atomic thick substance is absolutely elastic and transparent, such $2 \mathrm{D}$ materials have been attracted to the area of flexible electronics where the functions have not been perfectly demonstrated with conventional semiconductors yet. Although the charge carrier mobilities in the $2 \mathrm{D}$ semiconductors are much lower than that in graphene, the existence of the band gap, typically in a range of $1-2 \mathrm{eV}$, complements the properties of gapless graphene in electronic and photonic applications. For instance, field-effect transistors (FETs) based on ultrathin $\mathrm{MoS}_{2}, \mathrm{WS}_{2}, \mathrm{WSe}_{2}$, and GaSe show on/off ratios higher than $1 \times 10^{5}$ whereas graphene FETs typically have on/off ratios only at the level of about $10 .{ }^{3,5-7}$ In optoelectronic applications, such as photodetectors, the bandgap allows effective photocurrent generation as well as a significant reduction of the dark current, which leads to higher sensitivity. GaSe is a particularly interesting material for optoelectronic devices due to the bandgap at $\sim 2.0 \mathrm{eV}$ in multilayer with a remarkably small energy difference from the indirect to the direct bandgap $\left(\Delta E_{\mathrm{g}}\right):^{8-11}$ an indirect bandgap of $\sim 3.0 \mathrm{eV}$ with wider $\Delta E_{\mathrm{g}}$ in monolayer is significantly diminished with narrower $\Delta E_{\mathrm{g}}$ as the number of layers increases and, eventually, to be a likely direct bandgap in multilayer. Several GaSe-based photodetectors have been recently demonstrated at the ultraviolet and visible spectral ranges. ${ }^{12-17}$ Photodetectors fabricated from exfoliated $4 \mathrm{~nm}$ thick GaSe nanosheets reached photosensitivities as high as 2.8 $\mathrm{A} / \mathrm{W}$ at $254 \mathrm{~nm} .^{13}$ Even higher response at a range of $1 \times 10^{5}$ A/W was achieved under low incident optical power $(\sim 1 \times$ $10^{-3} \mathrm{~mW} / \mathrm{cm}^{2}$ ) at $532 \mathrm{~nm}$ with a hybrid graphene-GaSe nanosheet structure where the metal contacts were replaced by graphene to avoid the formation of Schottky contacts. ${ }^{13,18}$ Photoresponse in the broad wavelength range (from 400 to $2000 \mathrm{~nm}$ ) is further studied based on layer-by-layer growth $\mathrm{GaSe} / \mathrm{GaSb}$ heterostructure, demonstrating clear photoresponse in the near-infra (NIR) region with highest responsivity

Received: April 25, 2018

Accepted: July 31, 2018

Published: July 31, 2018 

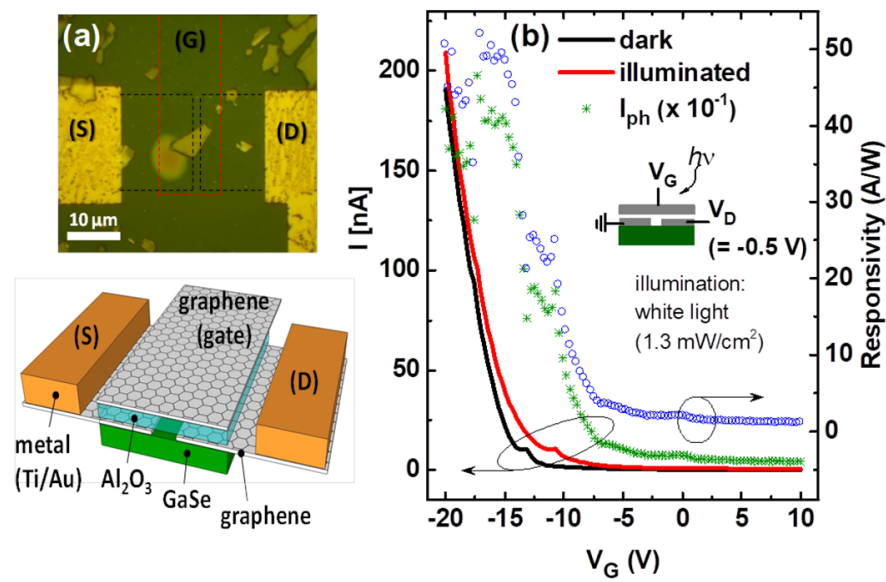

(c)
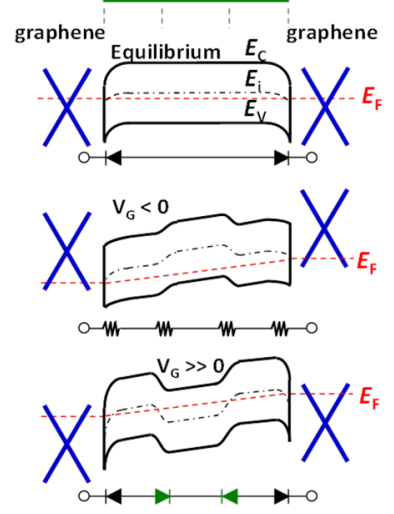

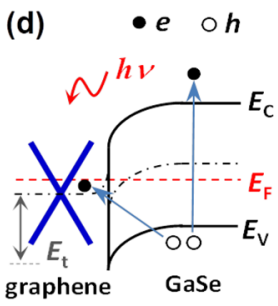

Figure 1. Device I. (a) Optical image and schematic of a graphene-gated GaSe FET with graphene contacts. Dashed lines in black and red denote a graphene contact and a topgate, respectively. The GaSe channel size is $1 \mu \mathrm{m} \times 4 \mu \mathrm{m}$ (length $\times$ width). (b) Transfer characteristics of the device measured in dark and under illumination (white light) at $V_{\mathrm{D}}=-0.5 \mathrm{~V}$. Plots with symbols indicate $I_{\mathrm{ph}}$ (green stars) and corresponding responsivity (blue circles). The level of $I_{\mathrm{ph}}$ should be divided by 10 for a real value. (c) Band diagrams with equivalent circuitries with components of diodes and resistors for the case of equilibrium $\left(V_{D}=0\right), V_{G}<0$, and $V_{G} \gg 0$ with negative $V_{D}$. Symbols in green denote the $\mathrm{p}-\mathrm{n}$ junction diodes in $\mathrm{GaSe}$. (d) Diagram describing the process of the electron-hole pair generation by illumination at the graphene-GaSe interface. $h \nu$ is the incident photon energy. $E_{\mathrm{C}}, E_{\mathrm{V}}, E_{\mathrm{i}}$, and $E_{\mathrm{F}}$ are the conduction band, valence band, intrinsic, and Fermi level, respectively. $e$ and $h$ stand for electron and hole, respectively.

of $0.09 \mathrm{~A} / \mathrm{W}$ at $1060 \mathrm{~nm} .{ }^{19}$ Recently, photodetection of graphene sandwiched GaSe based heterostructures, such as, $\mathrm{WS}_{2}-\mathrm{GaSe}$ and GaSe-InSe, have been also investigated in the wavelengths at $270-520 \mathrm{~nm} \cdot{ }^{20-22}$ In the studies comparably fast response time for rising and falling estimated in approximately milliseconds has been reported.

Although the Schottky junctions in semiconductor can promote the photoexcited charge separation at reverse bias, the high contact resistance associated with Schottky barriers typically limits device performance. In graphene the ability to control the Fermi level through external gate allows the type of the contact on semiconductor to be changed from Schottky to ohmic and even the polarity of the Schottky diode can be actively switched. ${ }^{23}$ Tunable graphene contacts on GaSe have been recently utilized in construction of a FET where the contacts, instead of the channel, are subjected to the field modulation. ${ }^{24}$ The same effect can also be utilized in other $2 \mathrm{D}$ material based photodetectors to enhance the photogenerated current by modulating the barrier height or the depletion region with the tunable graphene contacts. For example, $\mathrm{MoS}_{2}$ photodetectors have been demonstrated with graphene contacts, ${ }^{25-27}$ proving that the sensitivity can be higher than these with the metal contacts. ${ }^{28-30}$ In addition, graphene can serve as a transparent topgate electrode for photodetectors. ${ }^{31}$

$2 \mathrm{D}$ heterostructures also allow detection of photons with spatially indirect absorption based on the type II band misalignment. This has been recently demonstrated in $\mathrm{MoTe}_{2} / \mathrm{MoS}_{2}$ heterostructures as near-infrared (NIR) absorption, where the photocurrent is expected to arise because of the interband carrier transition between the valence band of $\mathrm{MoTe}_{2}$ and the conduction band of $\mathrm{MoS}_{2} .{ }^{32}$ This provides new alternatives to conventional semiconductors typically used as NIR photodetectors. In the case of the graphene-2D heterojunction, the photogenerated current through the interband gap transitions can further be modulated by the gate, implying that the sensitivity range of the photodetector can be tuned.
In this study, we demonstrate the detection of NIR light at $730 \mathrm{~nm}-1550 \mathrm{~nm}$ using graphene-gated graphene-GaSe heterojunction FETs. The structure with the graphene topgate covering both GaSe channel and graphene contacts is utilized to enable effective photocurrent generation from the heterojunctions.

\section{EXPERIMENTAL SECTION}

Device Fabrication. All the GaSe flakes were prepared by mechanical exfoliation on $\mathrm{SiO}_{2} / \mathrm{Si}$ substrate. The flakes on the substrate were expected to be placed as a natural p-type in a room ambient condition (as demonstrated in previous studies)..$^{1,7,24,33}$ To avoid the damage of $\mathrm{GaSe}$ in device fabrication (e.g., surface damage during graphene etching by $\mathrm{O}_{2}$ plasma) and to secure the stability of material in ambient condition, we selected multilayer GaSe flakes with thickness of $80-450 \mathrm{~nm}$ (thickness: $80 \mathrm{~nm}$ for device I, $\sim 400 \mathrm{~nm}$ for device II, and $\sim 450 \mathrm{~nm}$ for device III). To form a heterostructure, a CVD graphene film grown by photothermal $\mathrm{CVD}^{34}$ was transferred onto a GaSe flake and, subsequently, patterned with oxygen plasma to define the electrodes for source $(S)$ and drain (D).

$\mathrm{Ti} / \mathrm{Au}(2 / 50 \mathrm{~nm})$ metal lines were fabricated for the graphene electrodes to lead to the contact pads. Afterward, a 30 nm-thick $\mathrm{Al}_{2} \mathrm{O}_{3}$ layer was deposited on the graphene-GaSe structure by atomic layer deposition (ALD) to form a gate dielectric. A second layer of graphene was transferred on the dielectric layer and patterned to define a gate electrode. The graphene gate covers the channel as well as the contacts to have an effect on modulation of the charge carriers in both the channel and contacts (graphene electrodes) simultaneously by the gate filed.

From the different measurement setups, three similar devices were introduced to in this experiment: device I was utilized for FET performance measurement including the photoresponse in a white light; device II for photoresponsivity analysis for the lasers at 980 , 1330 , and $1550 \mathrm{~nm}$; and device III for response time analysis at 532, 980, 1330, and $1550 \mathrm{~nm}$.

Characterization. Device I: All DC measurements with white light exposure were performed at room temperature using semiconductor parameter analyzer (HP4155A) in ambient conditions. The lamp light was focused onto the devices through optics. An optical power of the light was measured with a power meter (Coherent LaserCheck). Device II: Device performances with the 
laser excitations were monitored using Keithley 2400 m. The lasers were irradiated through the optical fiber and power was accurately calibrated using a LaserStar dual-channel power meter (7Z01601) before excitation. The irradiation diameters of all lasers were set to about $60 \mu \mathrm{m}$. Device III: The sample was placed on the stage under the optics that were connected to the laser sources. The sources of 1550 and $1330 \mathrm{~nm}$ were connected to an erbium-doped fiber amplifier (CYFA-BO-b301). The output of the amplifier was then connected a chopper. By modifying the current of the amplifier, the incident power of these two wavelengths was set to $80 \mu \mathrm{W}$. The sources of 980 and $532 \mathrm{~nm}$ were connected to the chopper directly and each of the powers was set to 1 and $6 \mu \mathrm{W}$, respectively. For calibration, the incident power was directly measured through the $20 \times$ objective lens (numerical aperture NA= 0.4 ). The spot size was calculated from the equation defined by Laser spot diameter $=1.22 \lambda$ / NA. The electrical performances were monitored using two Keithley source meters and the time-resolved light on-off cycles was implemented through the control of the chopper by setting the time and number of circles.

\section{RESULTS AND DISCUSSION}

An optical image and a schematic of fabricated device are shown in Figure 1a (device I). The channel length (between two graphene contacts) and width are 1 and $4 \mu \mathrm{m}$, respectively (see Figure S1). When graphene contacts $\mathrm{GaSe}$, the electrons in graphene transfer to the GaSe surface (forming a dipole layer at the interface) due to the level of work function of $\mathrm{GaSe}$ $\left(W_{\mathrm{GaSe}} \approx 5.25 \mathrm{eV}\right)$ higher than that of graphene $\left(W_{\mathrm{G}} \approx 4.6\right.$ $\mathrm{eV}),{ }^{1,7}$ which results in the energy band of GaSe is bent down and a slight p-doping of graphene at the equilibrium. Consequently, in device architecture, two Schottky barriers are formed within back-to-back diode configuration at the interface (as depicted in Figure 1c) and they are tunable under the gate control (the details regarding to the band structure and theory are described in our previous study ${ }^{24}$ ).

Device characteristics with gate voltage $\left(V_{\mathrm{G}}\right)$ varying from -20 to $10 \mathrm{~V}$ at $V_{\mathrm{D}}=-0.5 \mathrm{~V}$ are shown in Figure $1 \mathrm{~b}$, exhibiting the on/off ratio as a FET higher than $1 \times 10^{3}$. In device operation with p-type doped GaSe, when $V_{\mathrm{G}}<0$, the Schottky barrier height $(\mathrm{SBH})$ is lowered due to the downshifted Fermi level of graphene. In addition, the hole carriers are accumulated in the surface of GaSe channel when $\mathrm{V}_{G}$ is lower than flat band voltage $\left(V_{\mathrm{FB}}\right)$ which is considered to be $-0.55 \mathrm{~V},{ }^{24}$ leading to an significant current flow through the device (illustrated with a band diagram in Figure 1c for $V_{\mathrm{G}}<$ $0)$. In contrast, by shifting $V_{\mathrm{G}}$ toward positive to the $V_{\mathrm{FB}}$, the $\mathrm{SBH}$ is increased and the carriers at the channel surface are depleted, leading the dramatic decrease of the current. When $V_{\mathrm{G}} \gg 0\left(>V_{\mathrm{FB}}\right)$, the $\mathrm{SBH}$ is further increased and hole carriers in the channel surface are inverted to electrons, forming additional potential barrier $(\mathrm{p}-\mathrm{n}$ junctions) at the boundary of the channel, which exhibits as face to face diode configuration (Figure $1 \mathrm{c}$ for $V_{\mathrm{G}} \gg 0$ ). Therefore, the channel current is close to zero as shown in Figure 1b. Energy band statuses under different $V_{\mathrm{G}}$ are presented in Figure 1c with equivalent circuitries.

To understand the basic rectification behavior of the device in dark, we have simulated the transfer characteristics $\left(I-V_{\mathrm{G}}\right)$. Importantly, we have assumed the existence of interface trapped charges, which might induce the Fermi level pinning (FLP). By assuming the interface trapped charge density, $D_{i t}$ ranging from zero to infinity, the impact of the FLP on electrical characteristics can be captured in the full range that goes from the Schottky to the Mott limit. In doing so, we have used the device model fully explained in previous works. ${ }^{24,35}$ In this model, the device operation can be understood by analyzing the electrical behavior of its equivalent circuit, which made of two back-to-back gate tunable diodes, referred to as $D_{1}$ and $D_{2}$, as shown in the inset of Figure $2 \mathrm{a}$. The current
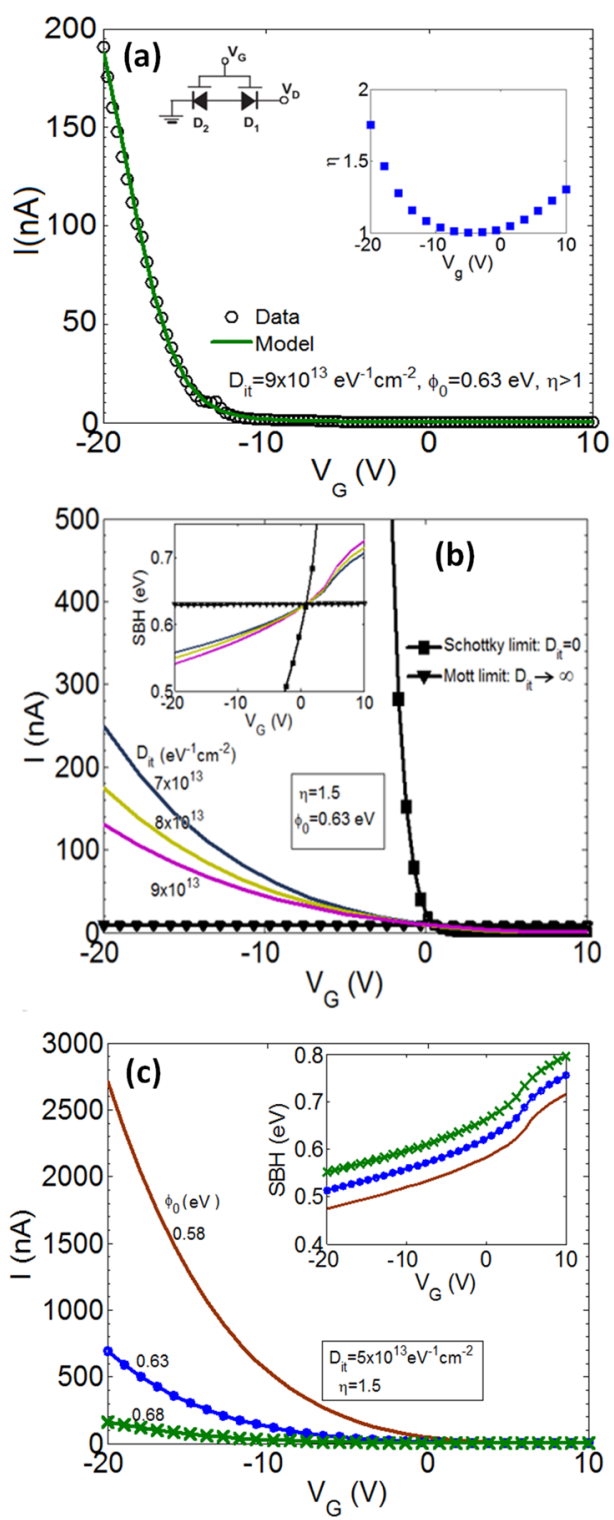

Figure 2. Simulated transfer characteristic of Device I at $V_{\mathrm{D}}=-0.5 \mathrm{~V}$. (a) Characteristics when $D_{\mathrm{it}}=9 \times 10^{13} \mathrm{eV}^{-1} \mathrm{~cm}^{-2}$ and $\phi_{0} \approx E_{\mathrm{g}} / 3$ are applied. Equivalent circuit for the device configuration and ideality factor depending on the gate voltage are shown in the inset. (b) Impact of the interface trapped charge density on the transfer characteristic of the device. The charge neutrality level $\left(\phi_{0}\right)$ was assumed to be $\sim 1 / 3$ of the GaSe bandgap. The SBH for holes as a function of $V_{G}$ is shown in the inset. Notice the insensitivity of the SBH to $V_{\mathrm{G}}$ in the Mott limit, where FLP dominates. (c) Impact of the charge neutrality level on the transfer characteristic in a partial FLP scenario. The expected SBH for holes is shown in the inset.

controlled by each of the gate tunable diodes have been computed with eq 1 . It follows the theory for the thermionic emission where the diode ideality factor $(\eta)$ affects both the current flow from the semiconductor to graphene and the reverse flow from graphene to the semiconductor: ${ }^{36}$ 

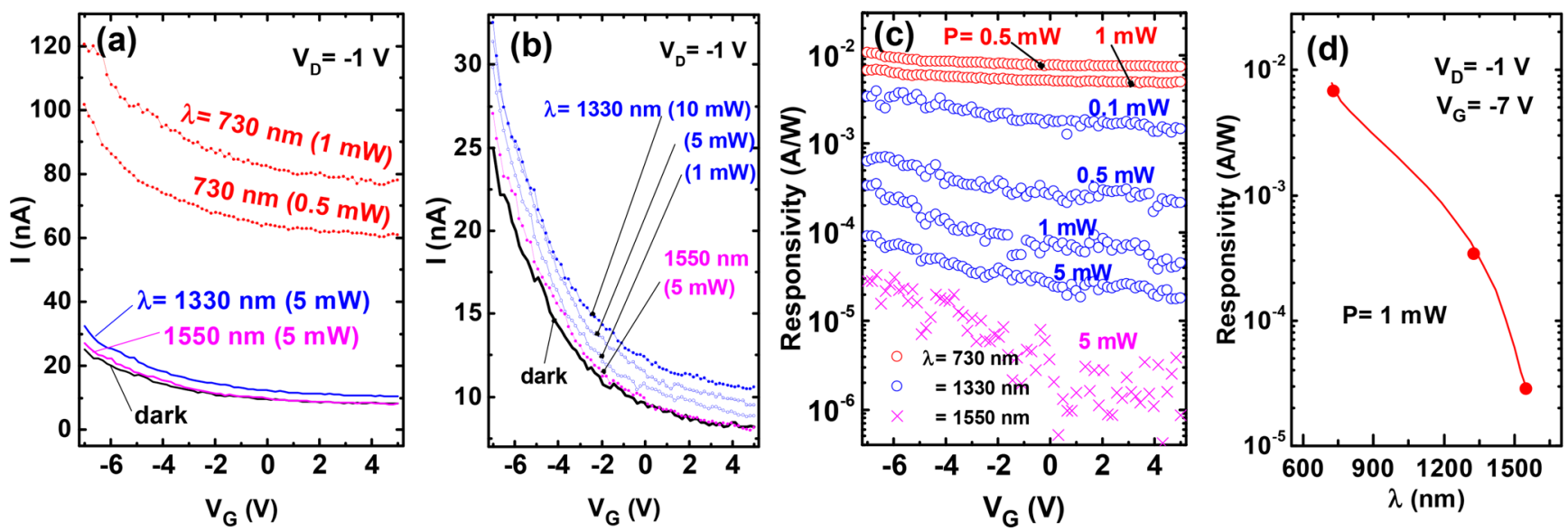

Figure 3. Wavelength-dependent photodetection of device II. (a) Transfer characteristics of the device under NIR illumination of $730 \mathrm{~nm}$ (red lines), $1330 \mathrm{~nm}$ (blue lines), and $1550 \mathrm{~nm}$ laser (pink line) and (b) with enlarged scale only for the 1330 and $1550 \mathrm{~nm}$ laser excitation with different optical powers. (c) Photoresponsivities for all NIR sources plotted in logarithmic scale. (d) Wavelength-dependent responsivity at $P=1$ $\mathrm{mW}$.

$$
I=A A^{*} T^{2} \exp \left(\frac{-q \phi_{b}}{k_{\mathrm{B}} T}\right) \exp \left(\frac{q V}{\eta k_{\mathrm{B}} T}\right)\left[1-\exp \left(\frac{-q V}{k_{\mathrm{B}} T}\right)\right]
$$

where $A, A^{*}$, and $T$ are the effective contact area between the graphene and the semiconductor, the Richardson's constant and the temperature, respectively. The term $\phi_{\mathrm{b}}$ is the SBH of the diode, $q$ the electric charge, and $k_{\mathrm{B}}$ the Boltzmann constant. $V$ refers to the applied bias. For the simulations we have used $A$ $=12 \mu \mathrm{m}^{2}, A^{*}=4.5 \times 10^{-6} \mathrm{~A} \mu \mathrm{m}^{-2} \mathrm{~K}^{-2}$, and $T=300 \mathrm{~K}$. The $\mathrm{SBH}$ has been computed by self-consistently solving the transport eq 1 together with the electrostatic equations governing the metal-oxide-graphene-semiconductor (MOGS) heterostructure, as detailed in refs 24 and 35. For the reader convenience the procedure has been schematized in Figure S2, where the band diagram of the MOGS heterostructure, corresponding to a vertical cut across the diode, can be also found. The simulation result for device I is plotted in Figure 2 (all parameters applied in this calculation are noted in Figure S2).

The bias was fixed with $V_{\mathrm{D}}=-0.5 \mathrm{~V}$ at $D_{1}$, so $D_{2}$ is reverse biased, and the current of the device is controlled by $D_{2}$. The best fit to the measurements in dark is reached when $D_{\text {it }} \approx 1 \times$ $10^{14} \mathrm{eV}^{-1} \mathrm{~cm}^{-2}$ and charge neutrality level $\left(\phi_{0}\right) \approx E_{\mathrm{g}} / 3$, where $E_{\mathrm{g}}$ is the band gap of $\mathrm{GaSe}$, are assumed. To gain consistency with the experiment, we have considered that the $\eta$ depends on the gate bias, as shown in the inset of Figure 2a. Impact of $D_{\text {it }}$ and $\phi_{0}$ on FET performance are also shown in Figure $2 \mathrm{~b}, \mathrm{c}$, respectively, with the corresponding $\mathrm{SBH}$ modulation for hole carriers of the controlling diode when the other parameters are fixed $(\eta=1.5)$. That scenario shown in Figure $2 \mathrm{c}$ corresponds to a partial FLP state that makes the $\mathrm{SBH}$ for holes quite insensitive to $V_{\mathrm{G}}$.

To investigate photoresponse behavior, we exposed the device to a white-light illumination. The light power $(P)$ was about $0.16 \mathrm{~mW}$ and the diameter of the illuminated area was about $4 \mathrm{~mm}$, leading to an average power intensity of $\sim 1.3$ $\mathrm{mW} / \mathrm{cm}^{2}$. The current measured under illumination $\left(I_{\text {illuminated }}\right)$ was compared with the current in dark $\left(I_{\text {dark }}\right)$ in transfer characteristics as shown in Figure $1 \mathrm{~b}$. The photogenerated current $\left(I_{\mathrm{ph}}=I_{\text {illuminated }}-I_{\text {dark }}\right)$ is observed in the all gate bias regime. The number of charge carriers is increased by electron-hole pair generation from the excitation of electrons

in the valence band by the photon impingement (as depicted in Figure 1d). They are separated by the applied $V_{\mathrm{D}}$, and eventually, the photogenerated current flows out through the terminals. In this measurement, $I_{\mathrm{ph}}$ was found varying from 20 $\mathrm{nA}$ to $0.4 \mathrm{nA}$ in the topgate voltage range of $-20 \mathrm{~V}<V_{\mathrm{G}}<10$ $\mathrm{V}$ at $V_{\mathrm{D}}=-0.5 \mathrm{~V}$. The corresponding photoresponsivity $(R)$ which is defined as the ratio of $I_{\mathrm{ph}}$ to the optical power incident to the channel area, i.e., $R=\frac{I_{\mathrm{ph}}}{P_{\text {channel }}}$, was calculated from 50 to 1 $\mathrm{A} / \mathrm{W}$ for white light $\left(P_{\text {illuminated }}=0.16 \mathrm{~mW}\right)$ as shown in Figure $1 \mathrm{~b}$ ( $P_{\text {channel }}=\frac{A_{\text {channel }}}{A_{\text {illuminated }}} P_{\text {illuminated }}$, where $P_{\text {channel }}$ is the power illuminated onto the channel and $A_{\text {channel }}$ and $A_{\text {illuminated }}$ are the area of the channel and the illumination). Although the channel size is defined to $1 \times 4 \mu \mathrm{m}^{2}$ by the graphene electrodes, we considered the whole GaSe flake $\left(7 \times 4 \mu \mathrm{m}^{2}\right)$ as a photoactive area when calculating photoresponsivity as the graphene electrodes are transparent. The measured responsivity is much higher than the device with metal contacts shown in Figure S-3 $\left(-12\right.$ to $2 \mathrm{~A} / \mathrm{W}$ at $\left.-6 \mathrm{~V}<V_{\mathrm{G}}<6 \mathrm{~V}\right)$.

To further examine the relation of photosensitivity to the wavelength $(\lambda)$ of light, the device was illuminated by three different monochromatic sources, i.e., 730, 1330, and $1550 \mathrm{~nm}$ lasers. Varied optical powers (i.e., $0.5-1 \mathrm{~mW}$ at $730 \mathrm{~nm}, 0.1-$ $10 \mathrm{~mW}$ at $1330 \mathrm{~nm}$, and $5 \mathrm{~mW}$ at $1550 \mathrm{~nm}$ ) were applied in our experiment. The diameters of all laser sources were set to be about $60 \mu \mathrm{m}$. Note that for this analysis another device, device II, having a structure exactly the same as device I was introduced because of the different experimental setup. The channel size is $1 \times 4 \mu \mathrm{m}^{2}$. Details in the architecture of device II can also be seen in Figure S4. Gate-dependent device characteristics under the laser excitation with varying laser powers are shown in Figure 3a, b (also see simulation for dark transfer characteristics in Figure S-5).

An obvious increase in $I_{\mathrm{DS}}$ with laser excitation was observed along the intensity of optical power (red curves for the $730 \mathrm{~nm}$, blue curves for the $1330 \mathrm{~nm}$, and pink curve for $1550 \mathrm{~nm}$ laser).

When applying $V_{\mathrm{D}}=-1 \mathrm{~V}$, maximum $I_{\mathrm{ph}}$ was $100 \mathrm{nA}$ for the $730 \mathrm{~nm}$ laser (with $P=1 \mathrm{~mW}$ ), $8 \mathrm{nA}$ for the $1330 \mathrm{~nm}$ laser (with $P=10 \mathrm{~mW}$ ), and $2 \mathrm{nA}$ for the $1550 \mathrm{~nm}$ laser (with $P=5$ $\mathrm{mW})$ at $V_{\mathrm{G}}=-7 \mathrm{~V}$. Corresponding photoresponsivities are plotted versus $V_{\mathrm{G}}$ as shown in Figure $3 \mathrm{c}$, presenting maximum 

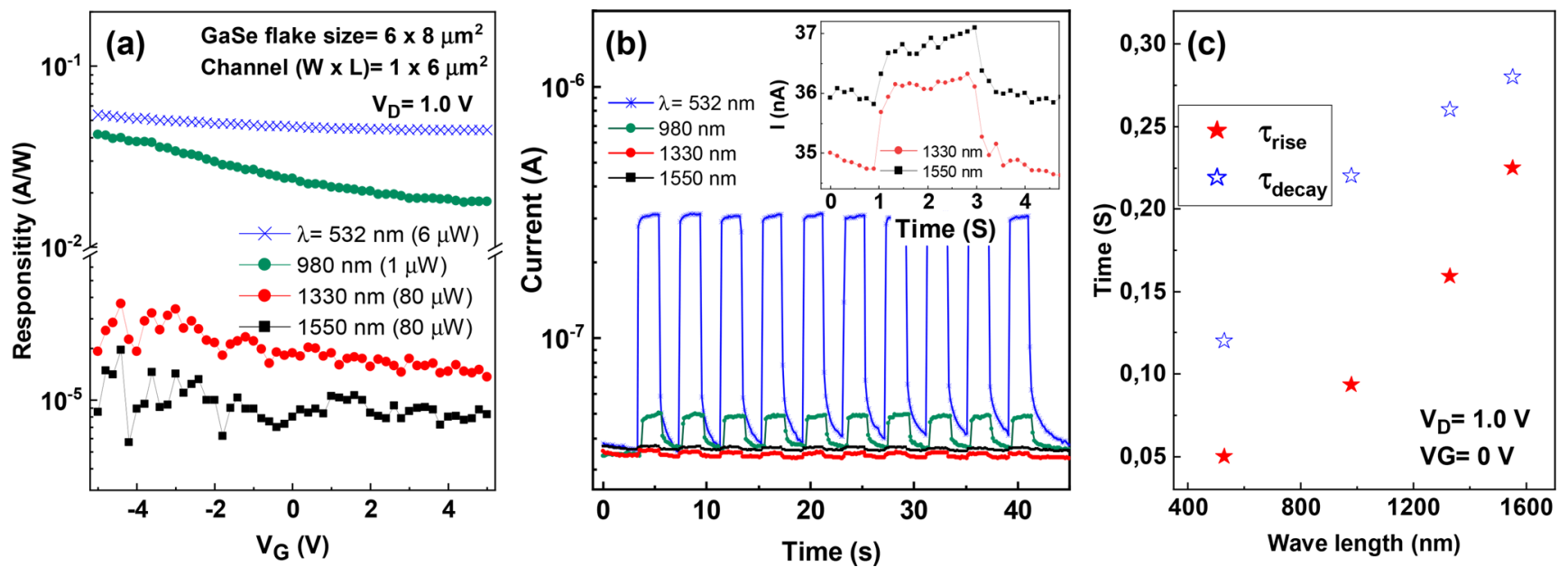

Figure 4. Device III. Photonic device behaviors under different laser excitations, 532, 980, 1330, and $1550 \mathrm{~nm}$. (a) Photoresponsivities for gate voltage. (b) Time-resolved photoresponse of the device. Enlarged scale of responses for the 1330 and $1550 \mathrm{~nm}$ are shown in the inset. (c) Time constants for the photocurrent rise $\left(\tau_{\text {rise }}\right)$ and decay $\left(\tau_{\text {decay }}\right)$ plotted to the wavelength.

responsivity as $10 \mathrm{~mA} / \mathrm{W}$ at $730 \mathrm{~nm}(P=0.5 \mathrm{~mW}), 3 \mathrm{~mA} / \mathrm{W}$ at $1330 \mathrm{~nm}(P=0.1 \mathrm{~mW})$, and $0.05 \mathrm{~mA} / \mathrm{W}$ at $1550 \mathrm{~nm}(P=5$ $\mathrm{mW}$ ) when $V_{\mathrm{G}}=-7 \mathrm{~V}$. For the relation to the wavelength, the maximum values of responsivity extracted at $P=1 \mathrm{~mW}$ were plotted with a trend line as seen in Figure $3 \mathrm{~d}$. In analysis, we have found that the responsivity in the heterostructure decreases exponentially at longer wavelengths in $\lambda=730-$ $1550 \mathrm{~nm}$. Note that the lower power light illumination leads to higher responsivity in calculation as the $\mathrm{I}_{\mathrm{ph}}$ does not increase for the power linearly but inverse exponentially as seen in Figure S6, where consequently, $R\left(=I_{\mathrm{ph}} / W\right)$ becomes smaller at the higher power. It is unclear why the increased rate of $I_{\mathrm{ph}}$ becomes smaller with the higher intensity; accordingly, further study is needed to clarify the relation. An optical switching ratio $\left(I_{\text {illuminated }} / I_{\text {dark }}\right)$ reached about 5 for the excitation at 730 $\mathrm{nm}(P=1 \mathrm{~mW}), 1.3$ at $1330 \mathrm{~nm}(P=10 \mathrm{~mW})$, and 1.1 at $1550 \mathrm{~nm}(P=5 \mathrm{~mW})$ under $V_{\mathrm{G}}=-7 \mathrm{~V}$.

Response time is an important figure of merit for the photodetector. To examine the response time another similar device (Device III) was introduced to the experiment. The flake size was $8 \times 6 \mu \mathrm{m}^{2}$ and the channel size (length $\times$ width) was $1 \times 6 \mu \mathrm{m}^{2}$, respectively (also see Figure S7). Structure of the device (graphene-gated graphene-GaSe heterojunction) was exactly the same with device I and II. Similarly to the previous measurements (for device I and II), different sources of lasers $(532,980,1330$, and $1550 \mathrm{~nm})$ were illuminated to the device to observe the device behaviors broadly. The photoresponsivities were calculated and they were exhibited from $\sim 1 \times 10^{2} \mathrm{~A} / \mathrm{W}$ to $\sim 1 \times 10^{-5} \mathrm{~A} / \mathrm{W}$ in the range of 532 to $1550 \mathrm{~nm}$ (Figure 4a). The levels are similar for device I and II as shown in Figures 1 and 3. Time responses of photocurrent are presented in Figure $4 b$, showing the current rise and fall corresponding to the laser on and off status. The rise and decay currents can be expressed as $I(t)=I_{\text {dark }}+A \exp \left(t / \tau_{\text {rise }}\right)$ and $I(t)$ $=I_{\text {dark }}+A \exp \left(-t / \tau_{\text {decay }}\right)$, respectively, where $t$ is the time, $A$ the scaling constant, and $\tau$ the time constant. ${ }^{13}$ The time constants were estimated by the curve-fit and identified as $\tau_{\text {rise }}$ $\left(\tau_{\text {decay }}\right)=0.05 \mathrm{~s}(0.12 \mathrm{~s})$ at $532 \mathrm{~nm}, 0.09 \mathrm{~s}(0.22 \mathrm{~s})$ at $980 \mathrm{~nm}$, $0.16 \mathrm{~s}(0.26 \mathrm{~s})$ at $1330 \mathrm{~nm}$, and $0.23 \mathrm{~s}(0.28 \mathrm{~s})$ at $1550 \mathrm{~nm}$, which indicates that the shorter wavelengths results in the faster photoresponse. To further evaluate the device performance, the photoconductive gain $(G)$ was calculated using the formula of $G=\tau / t_{\mathrm{L}}$, where $\tau$ is the carrier lifetime (here we used $\tau_{\text {decay }}$ extracted at the response time measurement for $\tau$ ) and $t_{\mathrm{L}}$ is the carrier transit time which is defined as $t_{\mathrm{L}}=L^{2}$ / $\mu V_{\mathrm{DS}}$ (where $L$ is the length of the channel and $\mu$ is the fieldeffect carrier mobility). The carrier mobility was extracted by the equation $=\frac{L}{W} \frac{\mathrm{d} I_{\mathrm{D}}}{\mathrm{d} V_{\mathrm{G}}} \frac{d}{\varepsilon} \frac{1}{V_{\mathrm{DS}}}$, where $W$ is the channel width, $d$ the thickness of dielectric layer, $\mathrm{Al}_{2} \mathrm{O}_{3}(d=40 \mathrm{~nm}), \varepsilon$ the dielectric constant, and $V_{\mathrm{DS}}$ the source-drain voltage. The carrier mobilities estimated from the transfer curve shown in Figure S7 were $\sim 2 \times 10^{-2}(532 \mathrm{~nm}), 8.2 \times 10^{-3}(980 \mathrm{~nm}), 4.6 \times$ $10^{-3}(1330 \mathrm{~nm})$, and $3.8 \times 10^{-3} \mathrm{~cm}^{2} /(\mathrm{V} \mathrm{s})(1550 \mathrm{~nm})$ at $V_{\mathrm{G}}=$ $-5 \mathrm{~V}$. From the calculations, $G$ was approximated to $2.5 \times 10^{5}$, $1.8 \times 10^{5}, 1.28 \times 10^{5}$, and $1.0 \times 10^{5}$ at 532, 980,1330, and $1550 \mathrm{~nm}$, respectively, under a gate voltage of $-5 \mathrm{~V}$.

The photocurrent arises at $\lambda=1330$ and $1500 \mathrm{~nm}$, even though their excitation energies of $\sim 0.93$ and $\sim 0.80 \mathrm{eV}$, respectively, are much lower than the bandgap of multilayer GaSe $(\sim 2.1 \mathrm{eV})$. This can be attributed to the surface-state absorption from interface-trapped charges or impurities possibly creating an allowed energy state in the forbidden energy gap. ${ }^{37}$ The mechanism may be also involved in interband transition between GaSe and graphene, which is in this case explained with an interlayer coupling interaction as the excited electrons in the valence band of GaSe transferring to the conduction band of graphene (intrinsic Fermi level) as claimed in a recent study of $2 \mathrm{D}-2 \mathrm{D}$ semiconductor heterojunctions. ${ }^{32}$ The interband transfer process with electron-hole pairs generated by the $h v$ is illustrated in Figure $1 \mathrm{~d}$. The interband energy $\left(E_{\mathrm{t}}=E_{\mathrm{v}}-E_{\mathrm{i} \text {, graphene }}\right)$ will be in $0.75-1.20 \mathrm{eV}$ when $E_{\mathrm{g}}, E_{\mathrm{v}}$, and $E_{\mathrm{i} \text {, graphene }}$ are considered to $1.97-2.1 \mathrm{eV},{ }^{1,8,38} 5.37-5.72 \mathrm{eV},{ }^{39,48}$ and $4.57 \pm 0.05 \mathrm{eV},^{41}$ respectively. Inversely, interband gap transition from $E_{\mathrm{i}, \text { graphene }}$ to $E_{\mathrm{c}}$ can possibly be occurred and its transition rate is slightly lower than that in $E_{\mathrm{i}, \text { graphene }}-E_{\mathrm{v}}$ due to the wider interband gap $(0.77-1.35 \mathrm{eV})$.

Although most of the 2D photodetectors have thus far been demonstrated for the visible spectral range, ${ }^{32,42,43}$ recently some 2D material-based devices have also been studied for the NIR range that has important applications (e.g., night vision and modern communication technologies). Performances of the photodetectors fabricated with representative $2 \mathrm{D}$ materials, 
Table 1. Comparison of Photodetectors Fabricated with Layered Materials $\left(\mathrm{GaSe}_{\mathrm{MoS}}, \mathrm{MoTe}_{2} \text {, and BP }\right)^{a}$

\begin{tabular}{|c|c|c|c|c|c|c|c|c|}
\hline ref & material and structure type & wavelength & power $\left(\mathrm{mW} / \mathrm{cm}^{2}\right)$ & $\left|V_{\mathrm{DS}}\right|(\mathrm{V})$ & $R(\mathrm{~A} / \mathrm{W})$ & $I_{\text {light }} / I_{\text {dark }}$ & gate control & $V_{\mathrm{G}}(\mathrm{V})$ \\
\hline 13 & $\mathrm{~m}(\mathrm{FL}) \mathrm{GaSe}$ & $254 \mathrm{~nm}$ & 1 & 5 & 2.8 & $\sim 25$ & $\mathrm{~N} / \mathrm{A}$ & $\mathrm{N} / \mathrm{A}$ \\
\hline 17 & $\mathrm{~m}(\mathrm{FL}) \mathrm{GaSe}$ & $410 \mathrm{~nm}$ & 0.01 & 8 & $\sim 5 \times 10^{3}$ & & $\mathrm{~N} / \mathrm{A}$ & $\mathrm{N} / \mathrm{A}$ \\
\hline 14 & ${ }^{c}(\mathrm{FL}) \mathrm{GaSe}$ & $405 \mathrm{~nm}$ & 50 & 10 & 0.017 & $1 \times 10^{3}$ & $\mathrm{~N} / \mathrm{A}$ & $\mathrm{N} / \mathrm{A}$ \\
\hline 15 & ${ }^{c}(\mathrm{FL}) \mathrm{GaSe}$ & halogen & 3.3 & 10 & 0.6 & $\sim 110$ & $\mathrm{~N} / \mathrm{A}$ & $\mathrm{N} / \mathrm{A}$ \\
\hline 16 & ${ }^{c}(\mathrm{FL}) \mathrm{GaSe}$ & white & 1.2 & 10 & 8.5 & $1 \times 10^{3}$ & BG & -60 \\
\hline 18 & ${ }^{\mathrm{c}} \mathrm{SLG}-{ }^{\mathrm{m}}(\mathrm{FL}) \mathrm{GaSe}$ & $532 \mathrm{~nm}$ & 1 & 1 & $\sim 1 \times 10^{4}$ & & BG & 0 \\
\hline 19 & $\mathrm{p}(\mathrm{FL}) \mathrm{GaSe}-\mathrm{p}(\mathrm{FL}) \mathrm{GaSb}$ & $1550 \mathrm{~nm}$ & $3 \times 10^{6}$ & 0 & $\sim 0.07$ & & $\mathrm{~N} / \mathrm{A}$ & $\mathrm{N} / \mathrm{A}$ \\
\hline 20 & ${ }^{\mathrm{c}} \mathrm{SLG}-{ }^{\mathrm{m}}(\mathrm{FL}) \mathrm{GaSe}-{ }^{\mathrm{m}}(\mathrm{FL}) \mathrm{WSe}_{2}-{ }^{\mathrm{m}} \mathrm{FLG}$ & $520 \mathrm{~nm}$ & $4 \times 10^{4}$ & 1.5 & 6.2 & & $\mathrm{~N} / \mathrm{A}$ & $\mathrm{N} / \mathrm{A}$ \\
\hline 44 & GaSe nanoribbons & $350 \mathrm{~nm}$ & 3.03 & 5 & 31.1 & 400 & $\mathrm{~N} / \mathrm{A}$ & $\mathrm{N} / \mathrm{A}$ \\
\hline \multirow[t]{2}{*}{27} & ${ }^{m} \mathrm{SLG}-{ }^{\mathrm{m}}(\mathrm{FL}) \mathrm{MoS}_{2}$ & $520 \mathrm{~nm}$ & 14 & 1 & $2 \times 10^{3}$ & $\sim 1 \times 10^{5}$ & BG & 0 \\
\hline & & $850 \mathrm{~nm}$ & 14 & 1 & $\sim 0$ & $\sim 0.5$ & BG & 50 \\
\hline 32 & ${ }^{\mathrm{m}}(\mathrm{FL}) \mathrm{MoTe}-{ }^{\mathrm{m}}(\mathrm{FL}) \mathrm{MoS}_{2}$ & $1550 \mathrm{~nm}$ & $2.5 \times 10^{5}$ & 0.8 & $\sim 1.6 \times 10^{-5}$ & $\sim 5.5$ & $\mathrm{~N} / \mathrm{A}$ & $\mathrm{N} / \mathrm{A}$ \\
\hline 42 & $\mathrm{~m}(\mathrm{ML}) \mathrm{BP}$ & $1550 \mathrm{~nm}$ & $3 \times 10^{6}$ & 0 & $5 \times 10^{-3}$ & & $\mathrm{~N} / \mathrm{A}$ & $\mathrm{N} / \mathrm{A}$ \\
\hline \multirow[t]{2}{*}{43} & $\mathrm{~m}(\mathrm{FL}) \mathrm{BP}$ & $1550 \mathrm{~nm}$ & & 0.4 & 0.14 & & $\mathrm{TG}$ & -8 \\
\hline & $\mathrm{m}(\mathrm{ML}) \mathrm{BP}$ & $1550 \mathrm{~nm}$ & & 2 & 0.66 & & $\mathrm{~N} / \mathrm{A}$ & $\mathrm{N} / \mathrm{A}$ \\
\hline \multirow[t]{3}{*}{ this work } & ${ }^{\mathrm{c}} \mathrm{SLG}-{ }^{\mathrm{m}}(\mathrm{ML}) \mathrm{GaSe}$ (Device II) & $730 \mathrm{~nm}$ & $1.8 \times 10^{4}$ & 1 & 0.01 & 5 & TG & -7 \\
\hline & & $1330 \mathrm{~nm}$ & $3.5 \times 10^{3}$ & 1 & 0.003 & 1.3 & & \\
\hline & & $1550 \mathrm{~nm}$ & $1.8 \times 10^{5}$ & 1 & $5 \times 10^{-5}$ & 1.1 & & \\
\hline
\end{tabular}

${ }^{a} \mathrm{FL}$, few layers (3-10 layers); ML, many layers (> $50 \mathrm{~nm}$ in thickness); SLG, single layer graphene; ${ }^{\mathrm{m}}$, mechanical chemical exfoliation: ${ }^{\mathrm{c}}, \mathrm{CVD}^{\mathrm{p}}{ }^{\mathrm{p}}$ physical vapor deposition (PVD); white, white light; BG, backgate; TG, topgate; and N/A, not applicable.

such as GaSe, $\mathrm{MoS}_{2}$, and $\mathrm{BP}$, including the structures combining with graphene or $\mathrm{MoTe}_{2}$, are listed in Table 1 to compare the capabilities for the NIR detection.

The comparison shows that the photoresponsivity is enhanced when a heterostructure is employed, for example, for $\mathrm{GaSe}$ as seen in refs 13-20. (although the device in ref 18 was characterized in a vacuum). Graphene- $\mathrm{MoS}_{2}$ heterojunction in ref 27 shows the remarkably high responsivity at 520-532 nm but almost no responsivity at $850 \mathrm{~nm}$. The absence of indirect transfer mediated response at NIR might be due to the $E_{\mathrm{t}}$ in 1.12-1.83 eV from the bandgap of monolayer $\mathrm{MoS}_{2}$ as $\sim 1.95 \mathrm{eV}$ and an electron affinity of $\mathrm{MoS}_{2}$ as 3.74$4.45 \mathrm{eV}^{45,46}$ For multilayer BP, a sensitivity for NIR range is more rigid as the bandgap of $\mathrm{BP}$ is predicted to $\sim 0.3 \mathrm{eV}$ in bulk ( $\sim 2.0 \mathrm{eV}$ in monolayer) as demonstrated in refs 42 and 43. $\mathrm{BP}$ is, however, known as a material highly sensitive to environment, especially for moisture. We here applied CVD graphene transferred onto the GaSe flakes using water-based technique and directly patterned the graphene under oxygen plasma without a protecting mask for $\mathrm{GaSe}$, which gives more freedom in device fabrication and integration.

\section{CONCLUSIONS}

We fabricated graphene-gated GaSe FETs and examined their electrical properties and photoresponse behaviors from visible to NIR. For the FET, graphene was employed both as a contact and a local topgate to allow the light transferring through the top-layers. In the experiment, the devices were characterized under illumination with white light and, particularly, three different monochromatic sources, i.e., 750 and $1330 \mathrm{~nm}$, and $1550 \mathrm{~nm}$ lasers. The maximum responsivity was observed as $10 \mathrm{~mA} / \mathrm{W}$ at $730 \mathrm{~nm}, 3 \mathrm{~mA} / \mathrm{W}$ at $1330 \mathrm{~nm}$, and $0.05 \mathrm{~mA} / \mathrm{W}$ at $1550 \mathrm{~nm}$, respectively. We expect that the photoresponse for the NIR arises from the interband transition in graphene-GaSe heterojunction, i.e., electron excited in the valence band of $\mathrm{GaSe}$ transferring to the intrinsic Fermi level of graphene. The result indicates that effective NIR photodetection is available in graphene-2D semiconductor hetero- junctions allowing to control its responsivity using external gate. The graphene-gated heterojunction structure shown here is compatible with conventional technologies in micro- and nanofabrication, providing more possibilities for monolithic device integration.

\section{ASSOCIATED CONTENT}

\section{S Supporting Information}

The Supporting Information is available free of charge on the ACS Publications website at DOI: 10.1021/acsanm.8b00684.

Material properties identified with optical microscope, Raman, and atomic force microscopy (AFM) and $I-V$ simulations with all parameters and equations utilized in calculations (PDF)

\section{AUTHOR INFORMATION}

\section{Corresponding Author}

*E-mail: sanna.arpiainen@vtt.fi.

ORCID $\odot$

Wonjae Kim: 0000-0001-7636-8360

Zhipei Sun: 0000-0002-9771-5293

\section{Notes}

The authors declare no competing financial interest.

\section{ACKNOWLEDGMENTS}

This project has received funding from the European Union's Horinon 2020 research and innovation programme under grant agreement No 785219. This, work was also partially funded by the Ministerio de Economía y Competitividad under the project TEC2015-67462-C2-1-R. The authors also acknowledge the funding from the Academy of Finland (Grants 276376, 284548, 295777, 304666, 312294, 312297, 312551, and 314810), TEKES-the Finnish Funding Agency for Technology and Innovation. The authors also thank Dr. Stephan Suckow in AMO GmbH for fruitful discussions about photonic device behavior. 


\section{REFERENCES}

(1) Bube, R. H.; Lind, E. L. Photoconductivity of Gallium Selenide Crystals. Phys. Rev. 1959, 115, 1159-1164.

(2) Fivaz, R.; Mooser, E. Mobility of Charge Carriers in Semiconducting Layer Structures. Phys. Rev. 1967, 163, 743-755.

(3) Radisavljevic, B.; Radenovic, A.; Brivio, J.; Giacometti, V.; Kis, A. Single-Layer MoS2 Transistors. Nat. Nanotechnol. 2011, 6, 147-150.

(4) Manzeli, S.; Ovchinnikov, D.; Pasquier, D.; Yazyev, O. V.; Kis, A. 2D Transition Metal Dichalcogenides. Nat. Rev. Mater. 2017, 2, 17033.

(5) Jo, S.; Ubrig, N.; Berger, H.; Kuzmenko, A. B.; Morpurgo, A. F. Mono- and Bilayer WS2 Light-Emitting Transistors. Nano Lett. 2014, 14, 2019-2025.

(6) Liu, W.; Kang, J.; Sarkar, D.; Khatami, Y.; Jena, D.; Banerjee, K. Role of Metal Contacts in Designing High-Performance Monolayer nType WSe2 Field Effect Transistors. Nano Lett. 2013, 13, 1983-1990.

(7) Late, D. J.; Liu, B.; Luo, J.; Yan, A.; Matte, H. S. S. R.; Grayson, M.; Rao, C. N. R.; Dravid, V. P. GaS and GaSe Ultrathin Layer Transistors. Adv. Mater. 2012, 24, 3549-3554.

(8) Aulich, E.; Brebner, J. L.; Mooser, E. Indirect Energy Gap in GaSe and GaS. Phys. Status Solidi B 1969, 31, 129-131.

(9) Cingolani, A.; Minafra, A.; Tantalo, P.; Paorici, C. Edge Emission in GaSe and GaS. Phys. Status Solidi 1971, 4, K83-K85.

(10) Mooser, E.; Schlüter, M. The Band-Gap Excitons in Gallium Selenide. Nuovo Cim.B 1973, 18, 164-208.

(11) Capozzi, V.; Montagna, M. Optical Spectroscopy of Extrinsic Recombinations in Gallium Selenide. Phys. Rev. B: Condens. Matter Mater. Phys. 1989, 40, 3182-3190.

(12) Kübler, C.; Huber, R.; Tübel, S.; Leitenstorfer, A. Ultrabroadband Detection of Multi-Terahertz Field Transients with GaSe Electro-Optic Sensors: Approaching the near Infrared. Appl. Phys. Lett. 2004, 85, 3360 .

(13) Hu, P.; Wen, Z.; Wang, L.; Tan, P.; Xiao, K. Synthesis of FewLayer GaSe Nanosheets for High Performance Photodetectors. ACS Nano 2012, 6, 5988-5994.

(14) Lei, S.; Ge, L.; Liu, Z.; Najmaei, S.; Shi, G.; You, G.; Lou, J.; Vajtai, R.; Ajayan, P. M. Synthesis and Photoresponse of Large GaSe Atomic Layers. Nano Lett. 2013, 13, 2777-2781.

(15) Zhou, Y.; Nie, Y.; Liu, Y.; Yan, K.; Hong, J.; Jin, C.; Zhou, Y.; Yin, J.; Liu, Z.; Peng, H. Epitaxy and Photoresponse of TwoDimensional GaSe Crystals on Flexible Transparent Mica Sheets. ACS Nano 2014, 8, 1485-1490.

(16) Li, X.; Lin, M.-W.; Puretzky, A. A.; Idrobo, J. C.; Ma, C.; Chi, M.; Yoon, M.; Rouleau, C. M.; Kravchenko, I. I.; Geohegan, D. B.; Xiao, K.; et al. Controlled Vapor Phase Growth of Single Crystalline, Two-Dimensional GaSe Crystals with High Photoresponse. Sci. Rep. 2015, 4, 5497.

(17) Cao, Y.; Cai, K.; Hu, P.; Zhao, L.; Yan, T.; Luo, W.; Zhang, X.; $\mathrm{Wu}, \mathrm{X}$.; Wang, K.; Zheng, H. Strong Enhancement of Photoresponsivity with Shrinking the Electrodes Spacing in Few Layer GaSe Photodetectors. Sci. Rep. 2015, 5, 8130.

(18) Lu, R.; Liu, J.; Luo, H.; Chikan, V.; Wu, J. Z. Graphene/GaSeNanosheet Hybrid: Towards High Gain and Fast Photoresponse. Sci. Rep. 2016, 6, 19161.

(19) Wang, P.; Liu, S.; Luo, W.; Fang, H.; Gong, F.; Guo, N.; Chen, Z.-G.; Zou, J.; Huang, Y.; Zhou, X.; et al. Arrayed Van Der Waals Broadband Detectors for Dual-Band Detection. Adv. Mater. 2017, 29, 1604439.

(20) Wei, X.; Yan, F.; Lv, Q.; Shen, C.; Wang, K. Fast Gate-Tunable Photodetection in the Graphene Sandwiched WSe 2 /GaSe Heterojunctions. Nanoscale 2017, 9, 8388-8392.

(21) Yan, F.; Zhao, L.; Patanè, A.; Hu, P.; Wei, X.; Luo, W.; Zhang, D.; Lv, Q.; Feng, Q.; Shen, C.; et al. Fast, Multicolor Photodetection with Graphene-Contacted $p$-GaSe/ $n$-InSe van Der Waals Heterostructures. Nanotechnology 2017, 28, 27LT01.

(22) Lv, Q.; Yan, F.; Wei, X.; Wang, K. High-Performance, SelfDriven Photodetector Based on Graphene Sandwiched GaSe/WS 2 Heterojunction. Adv. Opt. Mater. 2018, 6, 1700490.
(23) Yang, H.; Heo, J.; Park, S.; Song, H. J.; Seo, D. H.; Byun, K.-E.; Kim, P.; Yoo, I.; Chung, H.-J.; Kim, K. Graphene Barristor, a Triode Device with a Gate-Controlled Schottky Barrier. Science 2012, 336, $1140-1143$.

(24) Kim, W.; Li, C.; Chaves, F. A.; Jiménez, D.; Rodriguez, R. D.; Susoma, J.; Fenner, M. A.; Lipsanen, H.; Riikonen, J. Tunable Graphene-GaSe Dual Heterojunction Device. Adv. Mater. 2016, 28, $1845-1852$

(25) Roy, K.; Padmanabhan, M.; Goswami, S.; Sai, T. P.; Ramalingam, G.; Raghavan, S.; Ghosh, A. Graphene-MoS2 Hybrid Structures for Multifunctional Photoresponsive Memory Devices. Nat. Nanotechnol. 2013, 8, 826-830.

(26) Zhang, W.; Chuu, C.-P.; Huang, J.-K.; Chen, C.-H.; Tsai, M.-L.; Chang, Y.-H.; Liang, C.-T.; Chen, Y.-Z.; Chueh, Y.-L.; He, J.-H.; et al. Ultrahigh-Gain Photodetectors Based on Atomically Thin GrapheneMoS2 Heterostructures. Sci. Rep. 2015, 4, 3826.

(27) Rathi, S.; Lee, I.; Lim, D.; Wang, J.; Ochiai, Y.; Aoki, N.; Watanabe, K.; Taniguchi, T.; Lee, G.-H.; Yu, Y.-J.; et al. Tunable Electrical and Optical Characteristics in Monolayer Graphene and Few-Layer $\mathrm{MoS}_{2}$ Heterostructure Devices. Nano Lett. 2015, 15, 5017-5024.

(28) Yin, Z.; Li, H.; Li, H.; Jiang, L.; Shi, Y.; Sun, Y.; Lu, G.; Zhang, Q.; Chen, X.; Zhang, H. Single-Layer MoS2 Phototransistors. ACS Nano 2012, 6, 74-80.

(29) Lopez-Sanchez, O.; Lembke, D.; Kayci, M.; Radenovic, A.; Kis, A. Ultrasensitive Photodetectors Based on Monolayer MoS2. Nat. Nanotechnol. 2013, 8, 497-501.

(30) Fontana, M.; Deppe, T.; Boyd, A. K.; Rinzan, M.; Liu, A. Y.; Paranjape, M.; Barbara, P. Electron-Hole Transport and Photovoltaic Effect in Gated MoS2 Schottky Junctions. Sci. Rep. 2013, 3, 1634.

(31) Kim, W.; Li, C.; Chekurov, N.; Arpiainen, S.; Akinwande, D.; Lipsanen, H.; Riikonen, J. All-Graphene Three-Terminal-Junction Field-Effect Devices as Rectifiers and Inverters. ACS Nano 2015, 9, $5666-5674$.

(32) Zhang, K.; Zhang, T.; Cheng, G.; Li, T.; Wang, S.; Wei, W.; Zhou, X.; Yu, W.; Sun, Y.; Wang, P.; et al. Interlayer Transition and Infrared Photodetection in Atomically Thin Type-II $\mathrm{MoTe}_{2} / \mathrm{MoS}_{2}$ van Der Waals Heterostructures. ACS Nano 2016, 10, 3852-3858.

(33) Manfredotti, C.; Murri, R.; Rizzo, A.; Galassini, S.; Ruggiero, L. Deep Hole Traps in p-Type GaSe Single Crystals. Phys. Rev. B 1974, 10, 3387-3393.

(34) Riikonen, J.; Kim, W.; Li, C.; Svensk, O.; Arpiainen, S.; Kainlauri, M.; Lipsanen, H. Photo-Thermal Chemical Vapor Deposition of Graphene on Copper. Carbon 2013, 62, 43-50.

(35) Chaves, F. A.; Jimenez, D. The Role of the Fermi Level Pinning in Gate Tunable Graphene-Semiconductor Junctions. IEEE Trans. Electron Devices 2016, 63, 4521-4526.

(36) Sze, S. M. Semiconductor Devices: Physics and Technology, 2nd ed.; Wiley, 2014.

(37) Casalino, M.; Coppola, G.; Iodice, M.; Rendina, I.; Sirleto, L. Near-Infrared Sub-Bandgap All-Silicon Photodetectors: State of the Art and Perspectives. Sensors 2010, 10, 10571-10600.

(38) Jie, W.; Chen, X.; Li, D.; Xie, L.; Hui, Y. Y.; Lau, S. P.; Cui, X.; Hao, J. Layer-Dependent Nonlinear Optical Properties and Stability of Non-Centrosymmetric Modification in Few-Layer GaSe Sheets. Angew. Chem., Int. Ed. 2015, 54, 1185-1189.

(39) Ben Aziza, Z.; Henck, H.; Pierucci, D.; Silly, M. G.; Lhuillier, E.; Patriarche, G.; Sirotti, F.; Eddrief, M.; Ouerghi, A. Van Der Waals Epitaxy of GaSe/Graphene Heterostructure: Electronic and Interfacial Properties. ACS Nano 2016, 10, 9679-9686.

(40) Brudnyi, V. N.; Sarkisov, S. Y.; Kosobutsky, A. V. Electronic Properties of GaSe, InSe, GaS and GaTe Layered Semiconductors: Charge Neutrality Level and Interface Barrier Heights. Semicond. Sci. Technol. 2015, 30, 115019.

(41) Xia, F.; Farmer, D. B.; Lin, Y.-M.; Avouris, P. Graphene FieldEffect Transistors with High on/off Current Ratio and Large Transport Band Gap at Room Temperature. Nano Lett. 2010, 10, 715-718. 
(42) Engel, M.; Steiner, M.; Avouris, P. Black Phosphorus Photodetector for Multispectral, High-Resolution Imaging. Nano Lett. 2014, 14, 6414-6417.

(43) Youngblood, N.; Chen, C.; Koester, S. J.; Li, M. WaveguideIntegrated Black Phosphorus Photodetector with High Responsivity and Low Dark Current. Nat. Photonics 2015, 9, 247-252.

(44) Xiong, X.; Zhang, Q.; Zhou, X.; Jin, B.; Li, H.; Zhai, T. OneStep Synthesis of p-Type GaSe Nanoribbons and Their Excellent Performance in Photodetectors and Phototransistors. J. Mater. Chem. C 2016, 4, 7817-7823.

(45) Grubišíc Čabo, A.; Miwa, J. A.; Grønborg, S. S.; Riley, J. M.; Johannsen, J. C.; Cacho, C.; Alexander, O.; Chapman, R. T.; Springate, E.; Grioni, M.; et al. Observation of Ultrafast Free Carrier Dynamics in Single Layer $\mathrm{MoS}_{2}$. Nano Lett. 2015, 15, 5883-5887.

(46) Howell, S. L.; Jariwala, D.; Wu, C.-C.; Chen, K.-S.; Sangwan, V. K.; Kang, J.; Marks, T. J.; Hersam, M. C.; Lauhon, L. J. Investigation of Band-Offsets at Monolayer-Multilayer $\mathrm{MoS}_{2}$ Junctions by Scanning Photocurrent Microscopy. Nano Lett. 2015, 15, 2278-2284. 